\title{
Korrekter Umgang mit Abgeltungen für gleichwertige Gegenleistungen
}

Bruno Henggi ${ }^{a}$, Charlotte Schweizer ${ }^{b}$

a Verantwortlicher Public Affairs der FMH; ${ }^{b}$ Leiterin Abteilung Kommunikation der FMH

Am 1. Januar 2020 treten neue Vorschriften betreffend den Umgang mit Heilmitteln in Kraft. Die neuen Bestimmungen bringen strengere Regelungen mit sich, welche direkt für den Berufsalltag von Ärztinnen und Ärzten relevant sind. Sie sind in der Verordnung "Integrität und Transparenz im Heilmittelbereich (VITH)» festgehalten. Diese hat zum Ziel, die Integrität im Umgang mit Arzneimitteln zu stärken und dessen Transparenz zu erhöhen. Daher reguliert sie Preisrabatte und Abgeltungen seitens Arzneimittellieferanten stark und detailliert. Weiter enthält sie Bestimmungen über Unterstützungsbeiträge im Bereich Forschung sowie Weiter- und Fortbildung. Auch für die Annahme von Geschenken oder Einladungen zum Essen gibt es strikte Vorschriften.

Für Ärztinnen und Ärzte bedeutet dies, dass sie sich mit den Änderungen der neuen Verordnung vertraut machen müssen, um die neuen Bestimmungen achtsam und sorgfältig umsetzen zu können. Weiter müssen Ärztinnen und Ärzte ihre diesbezüglichen Handlungen konsequent und umfassend dokumentieren. Als Berufsverband erachtet es die FMH als ihre Pflicht, ihre Mitglieder auf die strengere Regelung aufmerksam zu machen und sie kontinuierlich und regelmässig über die neuen gesetzlichen Bestimmungen zu informieren. Dies möchten wir in anschaulicher Form in einer Artikelserie in vier Teilen realisieren.

Im vorliegenden Beitrag gehen wir näher auf Fragen ein, welche sich im Zusammenhang mit Abgeltungen für gleichwertige Gegenleistungen eines Arztes oder einer Ärztin stellen. Wir behandeln diese anhand von praktischen Beispielen, wie sie sich im Berufsalltag von Ärztinnen und Ärzten ergeben können.

\section{Abgeltung für gleichwertige Gegenleistungen}

Frage: Was beinhalten Abgeltungen für gleichwertige Gegenleistungen?

Antwort: Damit eine Abgeltung entsprechend der neuen Verordnung zulässig ist, muss eine gleichwertige Gegenleistung seitens der medizinischen Fachper- son - zum Beispiel einer Ärztin oder eines Arztes - erfolgen. Diese Gegenleistungen beim Einkauf von verschreibungspflichtigen Arzneimitteln können beispielsweise die Übernahme von Logistikaufwand, Lagerkosten oder Lagerrisiko beinhalten. Die Aufwände können entsprechend in Form von Mengenrabatten abgegolten werden. Auch Lehr-, Gutachtens- und Beratungstätigkeiten oder die Durchführung von wissenschaftlichen Studien und klinischen Versuchen können als adäquate und zulässige Gegenleistungen gelten. Denkbar sind weiter Praxiserfahrungsberichte, die in wissenschaftlich anerkannten Fachmedien publiziert werden können. Auch das Mitwirken in Beratungsgremien, Workshops oder an Marktforschungen kann abgegolten werden, soweit kein Werbezweck besteht.

Vorausgesetzt für die Zulässigkeit der Abgeltung der erwähnten Gegenleistungen ist erstens immer eine schriftliche Vereinbarung, aus welcher Art und Umfang von Gegenleistung und Abgeltung hervorgehen. Zweitens muss die Abgeltung in einem angemessenen Verhältnis zur Gegenleistung stehen.

Wichtig zu wissen ist auch, dass Leistungen, die eine Fachperson entweder für sich selbst erbringt, in Erfüllung gesetzlicher Verpflichtungen ausführt oder bereits anderweitig vergütet erhält, nicht mittels einer zusätzlichen Abgeltung entschädigt werden dürfen. 
Im Rahmen von sogenannten Fachgesprächen ist die Übernahme von Verpflegungskosten auch ohne schriftliche Vereinbarung zulässig, soweit die Verpflegungskosten für den Arzt oder die Ärztin CHF 100 nicht übersteigen. Wird dieser Betrag überschritten, ist auch für Verpflegungskosten während eines Fachgesprächs eine schriftliche Vereinbarung notwendig. Auch hier gilt, dass die Gegenleistung der Fachperson in einem angemessenen Verhältnis zu den Kosten der Verpflegung stehen muss. Es darf sich hierbei also nicht lediglich um eine Einladung zu einem Geschäftsessen handeln.

\section{Gleichwertigkeit der Abgeltung}

Frage: Wie ist festzustellen, ob eine Abgeltung gleichwertig ist mit der erbrachten Gegenleistung?

Antwort: Eine allgemeingültige Antwort kann hier nicht gegeben werden. Gefordert ist vorab der gesunde Menschenverstand. Wichtig ist die Angemessenheit des Verhältnisses zwischen Gegenleistung und Abgeltung. Fällt die Abgeltung aus dem Rahmen, sollte darauf verzichtet werden. Bei Grenzfällen besteht die Möglichkeit, beim Pharmaunternehmen bzw. bei der verantwortlichen Person eine Bestätigung für die Gleichwertigkeit einzuholen. Wird diese nicht erteilt, sollte aus Sicherheitsüberlegungen geprüft werden, eine solche Abgeltung abzulehnen.

\section{Onlinebestellungen}

Frage: Eine Firma hat entschieden, keine Rabatte mehr zu gewähren. Sie zahlt dem Leistungserbringer jedoch bei Onlinebestellungen 1\% als Gegenleistung. Ist diese Preisreduktion zulässig?

Antwort: Beim Preiserlass von $1 \%$ kann es sich um eine Abgeltung (der Lieferantin) für eine Gegenleistung (des Leistungserbringers) handeln, namentlich hinsichtlich der Vereinfachung des Bestellprozesses. Abgeltungen für Gegenleistungen müssen auf einer schriftlichen Vereinbarung beruhen und in einem angemessenen Verhältnis zueinander stehen, damit sie zulässig sind. Wir empfehlen, hierfür von der Firma einen schriftlichen Rahmenvertrag zu verlangen, mit dem die Firma die Gleichwertigkeit der Gegenleistung und Abgeltung bestätigt und der gleichzeitig die Gegenleistung (Onlinebestellung) umschreibt. Werden diese Voraussetzungen erfüllt, so sind solche Abgeltungen für Gegenleistungen von Ärztinnen und Ärzten zulässig.

Sollte die Firma die Gleichwertigkeit zwischen Gegenleistung und Abgeltung nicht bestätigen, oder wird keine schriftliche Vereinbarung abgeschlossen, so liegt eben gerade keine Abgeltung für gleichwertige Leistungen, sondern ein Preisrabatt vor. In solchen Fällen empfehlen wir, den erhaltenen Preisrabatt jeweils direkt an die Patientinnen und Patienten weiterzugeben oder den vollen Preis zu bezahlen.

\section{Skonto}

Frage: Eine Fachperson erhält für den Medikamenteneinkauf Skonto von 3\% bei Bezahlung der Rechnung innert 10 Tagen. Dürfen Ärztinnen und Ärzte diesen Preisnachlass annehmen?

Antwort: Dieser Skonto stellt grundsätzlich eine $\mathrm{Ge}$ genleistung dar: Ein Skonto bedeutet Liquidität für das Unternehmen sowie auch eine Reduzierung des Inkassorisikos. Für die Abgeltung von gleichwertigen Gegenleistungen ist allerdings ab dem 1. Januar 2020 neu eine schriftliche Vereinbarung vorausgesetzt. Weiter muss die Abgeltung gleichwertig sein, damit sie zulässig ist. Es stellt sich hier also die Frage, ob dies bei 3\% noch der Fall ist. Die Zulässigkeit der Höhe solcher Skonti muss künftig durch die Gerichte geklärt werden. Aus Vorsichtsgründen sollten Skonti einstweilen nicht höher als $2 \%$ angesetzt werden.

\section{Gesetzliche Grundlage VITH: Erläuterung}

Der Zweck der neuen gesetzlichen Grundlage ist zu vermeiden, dass die Verschreibung, Abgabe, Anwendung oder der Einkauf von verschreibungspflichtigen Arzneimitteln durch Zuwendungen jedweder Art beeinflusst wird. Anstatt ein vollständiges Verbot von Zuwendungen durchzusetzen, definiert die neue Verordnung einen Ausnahmekatalog, der abschliessend auflistet, was rechtlich weiterhin möglich sein soll. Darunter fallen auch Abgeltungen für gleichwertige Gegenleistungen, insbesondere für solche bei Bestellungen und Lieferungen von Heilmitteln. Die Gleichwertigkeit von Gegenleistungen und Abgeltungen muss mittels einer schriftlichen Vereinbarung festgehalten werden. Zudem muss sie nachvollziehbar und so präzise wie möglich beschrieben werden. Erfahrungen darüber, was die Behörden dereinst als gleichwertig einstufen werden, gibt es bisweilen noch keine. Die Behörde will sich namentlich an folgenden Richtlinien orientieren, welche in den Erläuterungen zur Verordnung genannt sind:

- Die Gegenleistung muss bezüglich Umfang und Aufwand dem Wert der Abgeltung ungefähr entsprechen.

- Die Abgeltung muss mittels separater Zahlung oder Verrechnung der Leistung erfolgen.

- Gegenleistungen im Rahmen der ordentlichen be- 
ruflichen Tätigkeit sind geringer zu entschädigen als jene, die einen Zusatzaufwand dieser Tätigkeit erfordern.

- Unzulässig sind Abgeltungen für Leistungen, die der Fachperson keinen zusätzlichen Aufwand verursachen. Damit sind Leistungen gemeint, die ohnehin erbracht werden müssen.

- Unzulässig sind auch Mehrfachabgeltungen, d.h., dass die Gegenleistung bereits anderweitig vergütet wurde, so zum Beispiel über den Lohn oder über die Rechnungsstellung an die Kundschaft oder die Versicherung.

- Nicht abgegolten werden darf Aufwand, der den Fachpersonen selbst direkten Nutzen bringt.

Vom Erfordernis der vorgängigen schriftlichen Vereinbarung ausgenommen ist die Übernahme von Verpflegungskosten durch ein Pharmaunternehmen im Rahmen eines Fachgesprächs. In diesem Fall darf der Gegenwert der Mahlzeit inklusive Getränke jedoch den Wert von 100 Franken nicht überschreiten.

\section{Art. 7 Abgeltungen für gleichwertige} Gegenleistungen

1 Als Abgeltungen für gleichwertige Gegenleistungen einer Fachperson oder Organisation nach Artikel 55 Absatz 2 Buchstabe c HMG zulässig sind Abgeltungen, die:

a. auf einer schriftlichen Vereinbarung basieren, woraus Art und Umfang von Gegenleistung und Abgeltung hervorgeht; und

b. in einem angemessenen Verhältnis zur Gegenleistung stehen.
2 Im Rahmen eines Fachgesprächs ist die Übernahme von Verpflegungskosten bis höchstens 100 Franken von der Verpflichtung nach Absatz 1 Buchstabe a ausgenommen.

3 Nicht abgegolten werden dürfen insbesondere Leistungen, die eine Fachperson oder Organisation:

a. für sich selbst erbringt;

b. in Erfüllung gesetzlicher Verpflichtungen erbringt; oder

c. anderweitig vergütet erhält.

4 Abgeltungen nach Absatz 1 sind insbesondere zulässig für:

a. Gegenleistungen beim Einkauf von verschreibungspflichtigen Arzneimitteln wie die Übernahme von Logistikaufwand, Lagerkosten oder Lagerrisiko;

b. Lehr-, Gutachtens- und Beratungstätigkeiten oder die Durchführung von wissenschaftlichen Studien und klinischen Versuchen;

c. Praxiserfahrungsberichte, die in einem wissenschaftlich anerkannten Fachmedium publiziert sind;

d. Mitwirkung in Beratungsgremien, Workshops oder an Marktforschungen, soweit kein Werbezweck besteht.

Wir empfehlen Ärztinnen und Ärzten, Vorsichtsmassnahmen zu treffen. Namentlich empfiehlt es sich:

- die Gleichwertigkeit von Gegenleistung und Abgeltung schriftlich bestätigen zu lassen;

- Vergütungen weiterzugeben, wenn keine Bestätigung der Gleichwertigkeit vorliegt. 